\title{
Electronic structure and magnetostrictive sensitivity of Fe-B metallic glasses
}

\author{
A. B. Beznosov, E. L. Fertman, V. V. Eremenko, and V. A. Desnenko \\ B. Verkin Institute for Low Temperature Physics and Engineering of the National Academy of Sciences of Ukraine \\ 47 Lenin Ave., Kharkov 61164, Ukraine \\ E-mail: beznosov@ilt.kharkov.ua \\ Received December 11, 2000
}

\begin{abstract}
The effect of mechanical tensile stresses $0 \leq \sigma \leq 62 \mathrm{MPa}$ on the low-field ( $0 \leq H \leq 45 \mathrm{Oe}$ ) magnetization curves of the metallic glasses $\mathrm{Fe}_{100-x} \mathrm{~B}_{x}(x=14,16,17,20)$ is studied at temperatures of 77 and $300 \mathrm{~K}$. The correlation between the electronic structure of the glasses and their magnetostrictive sensitivity $\kappa_{s}$ is revealed: the eutectic metallic glass $\mathrm{Fe}_{83} \mathrm{~B}_{17}$, which possesses a special electronic structure with a $30 \%$ lower conduction $(s p)$ electron density, has a value of $\kappa_{s}$ differing substantially from that of the other metallic glasses studied. Under low stresses $(0 \leq \sigma \leq 22 \mathrm{MPa})$ the eutectic alloy possesses a magnetostrictive sensitivity $\kappa_{s}\left(\sigma_{1}\right)$ which is higher than that of the rest of the alloys studied, while under high stresses (22 $\mathrm{MPa} \leq \sigma \leq 62 \mathrm{MPa}$ ) its magnetostrictive sensitivity $\kappa_{s}\left(\sigma_{2}\right)$ is close to zero.
\end{abstract}

PACS: 75.30.Cr, 75.30.Gw, 71.23.Cq

\section{Introduction}

In contrast to crystalline solids, whose atomic structures are precisely known thanks to $\mathrm{x}$-ray and neutron diffraction experiments, the precise atomic structure of amorphous solids is still unknown because of the comparatively low accuracy of the present-day technique for atomic structure determination of amorphous solids, particularly of metallic glasses [1]. Undoubtedly it can be said that amorphous solids consist of polyatomic ordered nanoclusters with disordered intercluster boundaries. Since the clusters have a certain atomic coordination, a monotonic variation of the chemical composition in metallic glasses results in a nonmonotonic variation of the atomic cluster structure, average binding energy per atom, and, as a consequence, their physical and mechanical properties [1-5]. In particular, it has been conjectured that metallic glasses of eutectic composition possess nanoclusters with quasicrystalline structure [6]. Our direct optical studies of the metallic glasses $\mathrm{Fe}_{100-x} \mathrm{~B}_{x}$ $(x=14,16,17,20)$ have established that the eutectic alloy $\mathrm{Fe}_{83} \mathrm{~B}_{17}$ possesses a special electronic structure with a $30 \%$ lower conduction electron concentration $[1,4]$. The difference in the electronic structures of the above-mentioned metallic glasses must obviously be reflected in their magnetic characteristics.
The present paper is devoted to a study of the effects of low mechanical stresses $0 \leq \sigma \leq 68 \mathrm{MPa}$ on the low-field $(0 \leq H \leq 45$ Oe $)$ magnetization curves $B(H)$ of the metallic glasses $\mathrm{Fe}_{100-x} \mathrm{~B}_{x}$ $(x=14,16,17,20)$ at temperatures of 77 and $300 \mathrm{~K}$. The goal is to determine the magnetostrictive sensitivity $\kappa_{s}=\partial B / \partial \sigma$ at low tensile stresses (here $B$ is the magnetic induction). This characteristic is of considerable applied interest, since metallic glasses have a wide field of applications, and in some cases a low magnetostrictive sensitivity is necessary (e.g., recording heads), while for other purposes (e.g., stress sensors) a high one is required [7].

\section{Experimental}

The $\mathrm{Fe}_{100-x} \mathrm{~B}_{x}(x=14,16,17,20)$ metallic glass samples were closed rings $45 \mathrm{~mm}$ in diameter, prepared from amorphous ribbons $30 \mu \mathrm{m}$ thick and $6 \mathrm{~mm}$ wide, obtained by the planar flow casting method. The effect of tension on the initial magnetic induction curve $B(H)$ was measured by the pulse-induction technique using a longitudinal magnetic field. Longitudinal stresses of 22 and $62 \mathrm{MPa}$ were created by the tension of the rings, which were placed in a thermostat. The magnetostrictive sensitivities $\kappa_{s}\left(\sigma_{1}\right)$ and $\kappa_{s}\left(\sigma_{2}\right)$ were obtained, corre- 
sponding to the average values in the tension ranges 0-22 MPa and 22-62 MPa, respectively.

The magnetic induction $B=(1+4 \pi \chi) H$ at arbitrary points of the magnetic field range were obtained using the following interpolation formulas for the magnetic susceptibility:

$$
\chi=\chi_{0} \frac{1+\xi \tanh \left[\xi^{-1}\left(H / H_{a}\right)^{q}+\left(H / H_{e}\right)^{p}\right]}{1+\left(H / H_{a}\right)^{q}}
$$

which describes the domain system reconstruction and better fits the «low-field» region, and

$$
\chi=\chi_{0} \frac{\xi \tanh \left[\xi^{-1} H^{0.5}+\left(H / H_{e}\right)^{p}\right]}{H^{0.5}+H / H_{a}},
$$

which has the correct «high-field» limit, reflecting the action of local anisotropy due to the zero-dimensional defect system, and better fits the magnetization reversibility region. The values of $\xi, p, q, H_{a}$, $H_{e}$, and $\chi_{0}$ were determined by fitting the experimental data. The parameters $\xi, p, H_{a}$, and $H_{e}$ in (1) differ from those in (2).

\section{Results and discussion}

\subsection{Initial susceptibility}

The initial susceptibility $\chi_{0}$ is determined by the spontaneous magnetization and anisotropy constants $[8,9]$. Both of these parameters are intimately related to the state character of the «magnetic» electron shells in the system. In this case these are the $3 d$-electron shells of the Fe ions in the alloys. The electrons of these shells (at least some of them) are quasilocalized and form narrow energy bands. Upon alloying, a fraction of the conduction $(s p$ ) electrons of the Fe subsystem is spent on the creation of valence bonds with the boron, so we can expect a certain component linear in the boron concentration $x$ in the concentration dependence of the $s p$-electron density in the system. Taking into account the expected reconstruction of the electron configuration of the Fe ions in the eutectic alloy, the concentration dependence of the $s p$-electron density $N_{s p}$ of the $\mathrm{Fe}-\mathrm{B}$ metallic glasses in the interval $14 \leq x \leq 21$ can be written in the form [4]

$$
N_{s p}(x)=n_{0}-\zeta x-v P(x),
$$

where $P(x)=\exp \left(-(x-0.17)^{2} / \delta^{2}\right)$ is the probability of the change of electronic configuration of the $\mathrm{Fe}$ ion. A similar dependence is realized for the

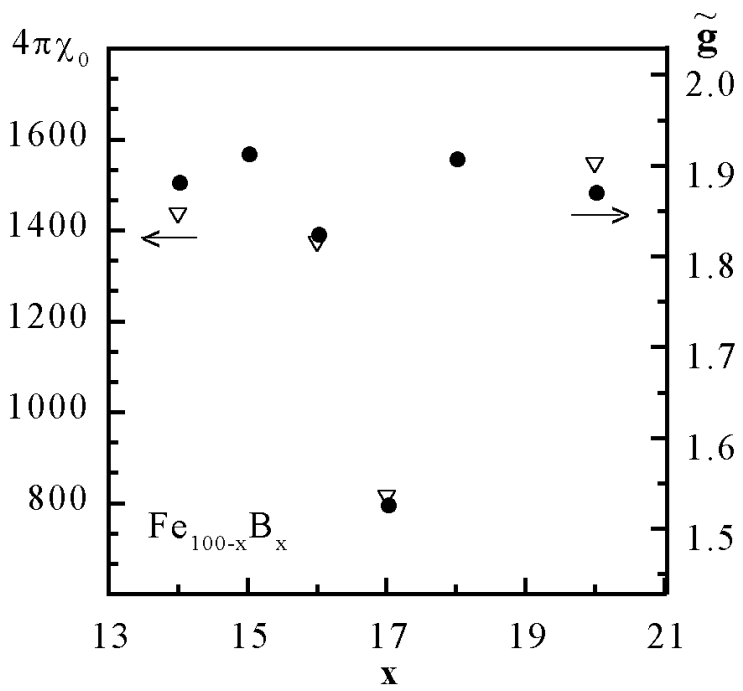

Fig. 1. The initial magnetic susceptibility $\chi_{0}(\nabla)$ and the «initial» (i.e., corrected by compensation of the linear part of concentration dependence) $g$ factor [3] ( $\bullet$ ) of the metallic glasses $\mathrm{Fe}_{100-x} \mathrm{~B}_{x}(x=14,16,17,20)$ versus $x$.

Landé $g$ factor, whose value also depends on the states of the «magnetic» electrons shells:

$$
g(x)=g_{0}+\eta x-\xi P(x) .
$$

Here the «initial» values $n_{0}$ and $g_{0}$, as well as $\zeta$, $v, \xi$, and $\delta$ are adjustable parameters.

The concentration dependence of the initial susceptibility $\chi_{0}$ and the «initial» Landé factor $\tilde{g}_{0}(x)=g(x)-\eta x$ is presented in Fig. 1, and Fig. 2 presents the concentration dependence of $\tilde{n}_{0}(x)=N_{s p}(x)+\zeta x$ and saturation magnetization $M_{s}$ ( $N_{s p}$ was derived from the optical ellipsometry data [4] in the three-parameter Drude model [10],

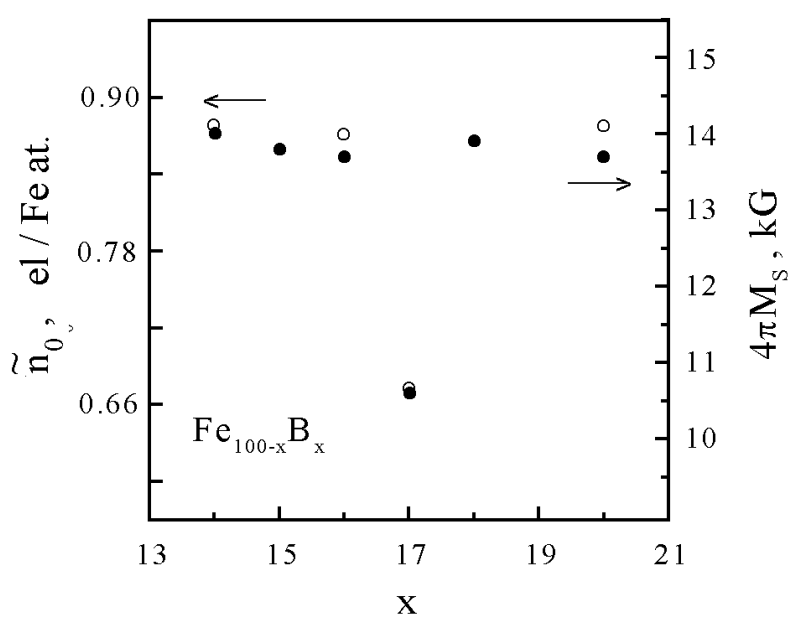

Fig. 2. Effective «initial» (i.e., corrected by compensation of the linear part of concentration dependence) conduction electron density $n_{0}(\bigcirc)$ and the low-temperature high-field saturation magnetization $M_{s}[3](\bullet)$ of the $\mathrm{Fe}_{100-x} \mathrm{~B}_{x}(x=14,16$, 17, 20) metallic glasses versus $x$. 


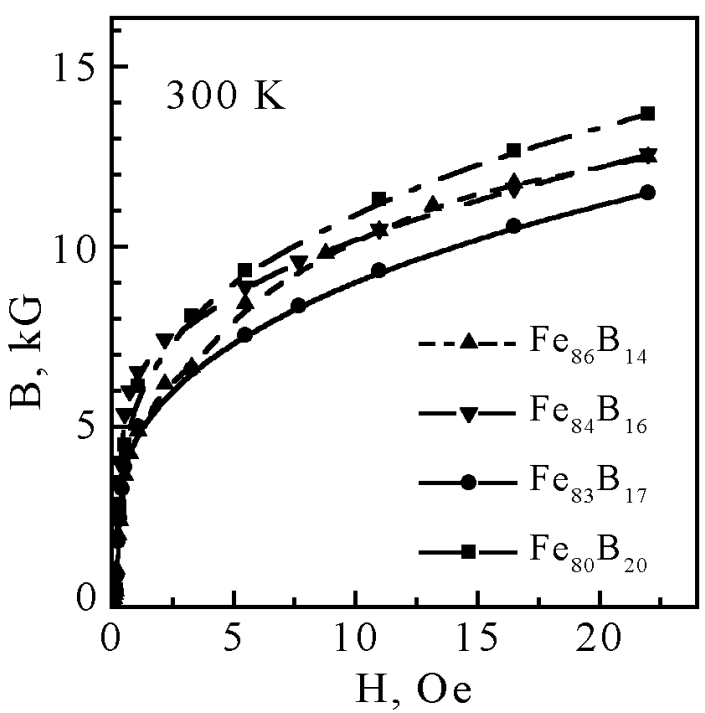

Fig. 3. The initial magnetization curves $B(H)$ of the $\mathrm{Fe}_{100-x} \mathrm{~B}_{x}$ $(x=14,16,17,20)$ metallic glasses at room temperature.

and $g_{0}$ and $M_{s}$ are obtained from the ferromagnetic resonance data in Ref. 3 ).

As can be seen from Figs. 1 and 2, there is a singularity in all the dependences at the point $x=17$. This indicates a substantial difference in the electronic structure of the eutectic metallic glass and the other compositions studied. One can assume that the states of the $\mathrm{Fe}$ ions in the eutectic glass are close to $\mathrm{Fe}^{2+}$, while the states of the $\mathrm{Fe}$ ions in the other alloys are close to $\mathrm{Fe}^{3+}$, as far as it is possible to speak about the valence in the case of such complex interatomic bonds as are realized in the transition metals.

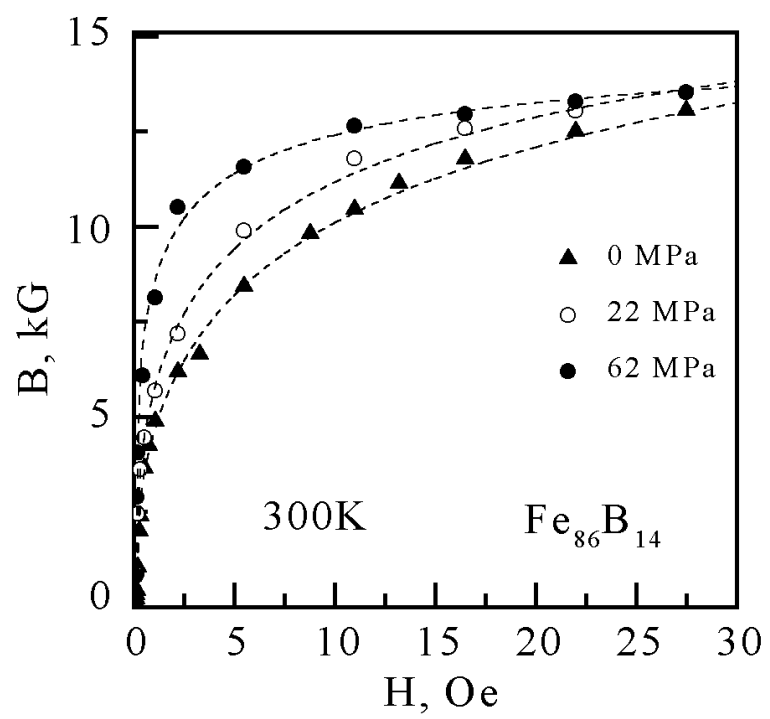

Fig. 4. The initial magnetization curves $B(H)$ of $\mathrm{Fe}_{86} \mathrm{~B}_{14}$ metallic glass under tensile stresses of 0,22 and $62 \mathrm{MPa}$ at room temperature.

\subsection{Magnetizing and tension effects}

The initial magnetization curves $B(H)$ for all the metallic glasses studied look similar (Fig. 3), but the eutectic alloy $\mathrm{Fe}_{83} \mathrm{~B}_{17}$ possesses the lowest value of $B$ in fields above 5 Oe at all the studied temperatures and stresses. The $B(H)$ curve for the eutectic alloy suggests that this alloy has the smallest saturation induction. This result corresponds to the saturation magnetization measurements in the paper [3] (compare Figs. 2 and 3).

The metallic glasses studied have a high positive magnetostriction, and applied tensile stresses lead to a growth in the magnetic induction $B$ and magnetic susceptibility $\chi$. The growth of the magnetic induction is mainly seen in fields up to about 30 Oe (Fig. 4). At both room and liquid nitrogen temperatures the growth of the maximum value of the magnetic susceptibility $\chi_{\max }$ of all the alloys under load is very sharp and range up to $1.5-3$ times at $62 \mathrm{MPa}$. This can be seen at Fig. 5, where the load effect is shown for the alloy $\mathrm{Fe}_{86} \mathrm{~B}_{14}$.

\subsection{Magnetostrictive sensitivity}

The field dependences of the magnetostrictive sensitivity $\kappa_{s}\left(\sigma_{1}\right)$ and $\kappa_{s}\left(\sigma_{2}\right)$ in the tensile stresses ranges $0 \leq \sigma \leq 22 \mathrm{MPa}$ and $22 \mathrm{MPa} \leq \sigma \leq 62 \mathrm{MPa}$, respectively for all the alloys studied have two maxima, one of which is located in the magnetic field region up to 0.5 Oe (Fig. 6) and the other one in the field range 1-11 Oe (Fig. 7). The values at the maxima $\kappa_{s 2 m}$ and their positions $H_{2 m}$ are shown in Table.

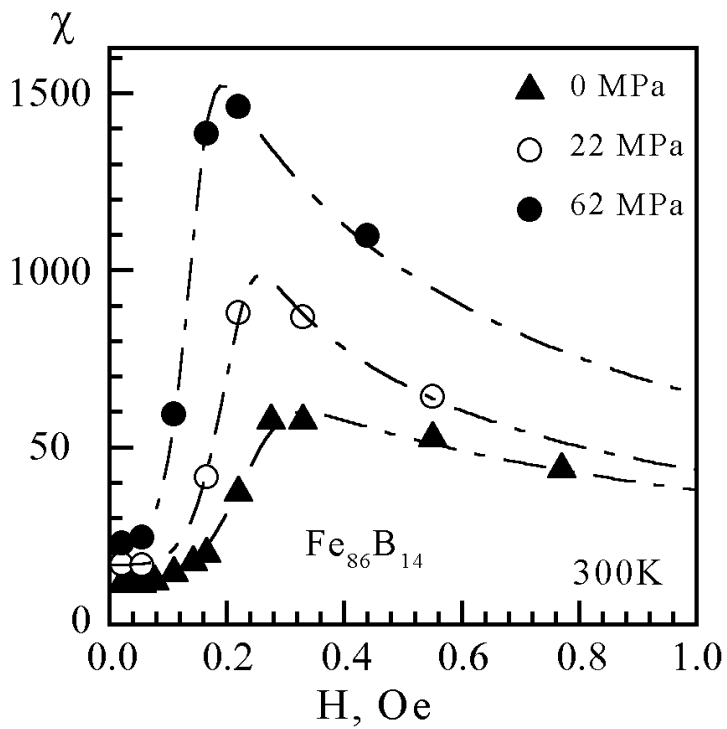

Fig. 5. Magnetic susceptibility $\chi$ of the $\mathrm{Fe}_{86} \mathrm{~B}_{14}$ metallic glass at room temperature under tensile loads of 0,22 and $62 \mathrm{MPa}$ versus magnetic field $H$. 
Characteristics of the magnetostrictive sensitivity $\kappa_{s}$ of metallic glasses $\mathrm{Fe}-\mathrm{B}$ at nitrogen $(77 \mathrm{~K})$ and room $(300 \mathrm{~K})$ temperatures and at average tensile stresses $\langle\sigma\rangle$ of $11 \mathrm{MPa}(0 \leq \sigma \leq 22 \mathrm{MPa})$ and $42 \mathrm{MPa}(22 \mathrm{MPa} \leq \sigma \leq 62 \mathrm{MPa})$, respectively. $H_{2 m}$ is the position of the second maximum $\kappa_{s 2 m}$ of the magnetostrictive sensitivity, and the average value $\left\langle\kappa_{s}\right\rangle$ corresponds to the range $0 \leq H \leq 35$ Oe of the magnetic field

\begin{tabular}{|c|c|c|c|c|c|c|c|c|c|c|c|c|}
\hline \multirow{3}{*}{ Alloy } & \multicolumn{4}{|c|}{$H_{2 m}$, Oe } & \multicolumn{4}{|c|}{$\kappa_{s 2 m}, \mathrm{G} / \mathrm{MPa}$} & \multicolumn{4}{|c|}{$\left\langle\kappa_{s}\right\rangle, \mathrm{G} / \mathrm{MPa}$} \\
\hline & \multicolumn{2}{|c|}{$\langle\sigma\rangle=11 \mathrm{MPa}$} & \multicolumn{2}{|c|}{$\langle\sigma\rangle=42 \mathrm{MPa}$} & \multicolumn{2}{|c|}{$\langle\sigma\rangle=11 \mathrm{MPa}$} & \multicolumn{2}{|c|}{$\langle\sigma\rangle=42 \mathrm{MPa}$} & \multicolumn{2}{|c|}{$\langle\sigma\rangle=11 \mathrm{MPa}$} & \multicolumn{2}{|c|}{$\langle\sigma\rangle=42 \mathrm{MPa}$} \\
\hline & $77 \mathrm{~K}$ & $300 \mathrm{~K}$ & $77 \mathrm{~K}$ & $300 \mathrm{~K}$ & $77 \mathrm{~K}$ & $300 \mathrm{~K}$ & $77 \mathrm{~K}$ & $300 \mathrm{~K}$ & $77 \mathrm{~K}$ & $300 \mathrm{~K}$ & $77 \mathrm{~K}$ & $300 \mathrm{~K}$ \\
\hline $\mathrm{Fe}_{86} \mathrm{~B}_{14}$ & 5.5 & 5.5 & 2.2 & 2.2 & 81 & 66 & 94 & 81 & 52 & 34 & 28 & 16 \\
\hline $\mathrm{Fe}_{84} \mathrm{~B}_{16}$ & 2.2 & 2.2 & 5.5 & 5.5 & 91 & 47 & 34 & 37 & 32 & 14 & 25 & 25 \\
\hline $\mathrm{Fe}_{83} \mathrm{~B}_{17}$ & 2.2 & 1.1 & 5.5 & 3.3 & 62 & 110 & 44 & 9 & 36 & 58 & 31 & 3 \\
\hline $\mathrm{Fe}_{80} \mathrm{~B}_{20}$ & 11 & 4.3 & 1.7 & 3.3 & 42 & 50 & 4 & 29 & 33 & 31 & 22 & 15 \\
\hline
\end{tabular}

As expected, the field dependences of the magnetostrictive sensitivity of the amorphous eutectic alloy $\mathrm{Fe}_{83} \mathrm{~B}_{17}$ differ substantially from those of other alloys (compare Figs. 7). Under low stresses in the whole interval of magnetic fields studied this alloy possesses a magnetostrictive sensitivity $\kappa_{s}\left(\sigma_{1}\right)$ higher than that for the other amorphous alloys studied. Under the higher stresses its magnetostrictive sensitivity $\kappa_{s}\left(\sigma_{2}\right)$ is weakly dependent on the field and is much lower (practically is equal to zero) than the other's (Figs. 7, 8, Table). No other alloy studied possesses such a difference between its magnetostrictive sensitivities at different loads (Fig. 8).

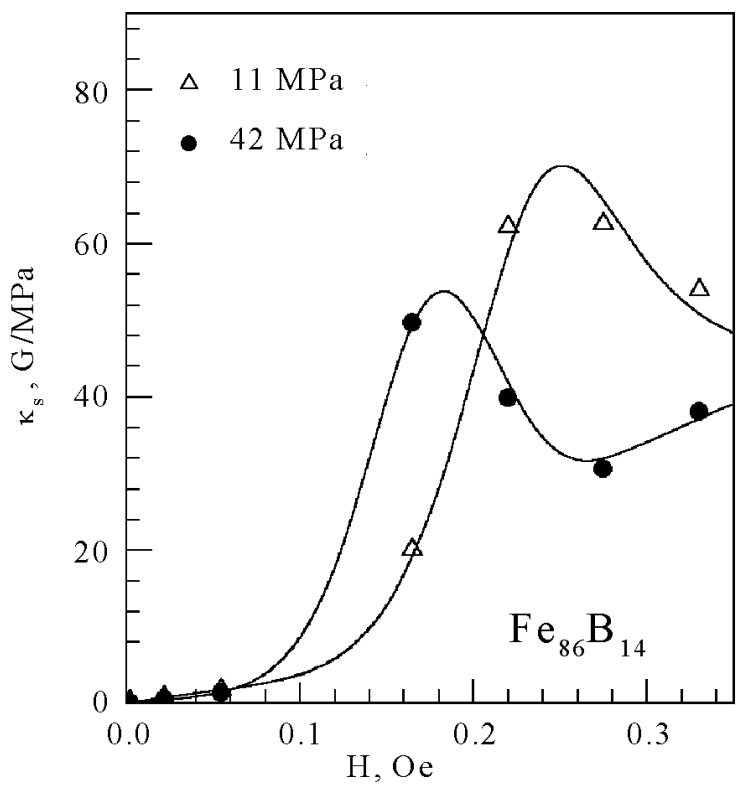

Fig. 6. The initial region of the field dependence of the magnetostrictive sensitivity $\kappa_{s}$ of the metallic glass $\mathrm{Fe}_{86} \mathrm{~B}_{14}$ in the tensile stress regions $0-22 \mathrm{MPa}(\langle\sigma\rangle=11 \mathrm{MPa})$ and 22-62 $\mathrm{MPa}$ $(\langle\sigma\rangle=42 \mathrm{MPa})$ at room temperature.
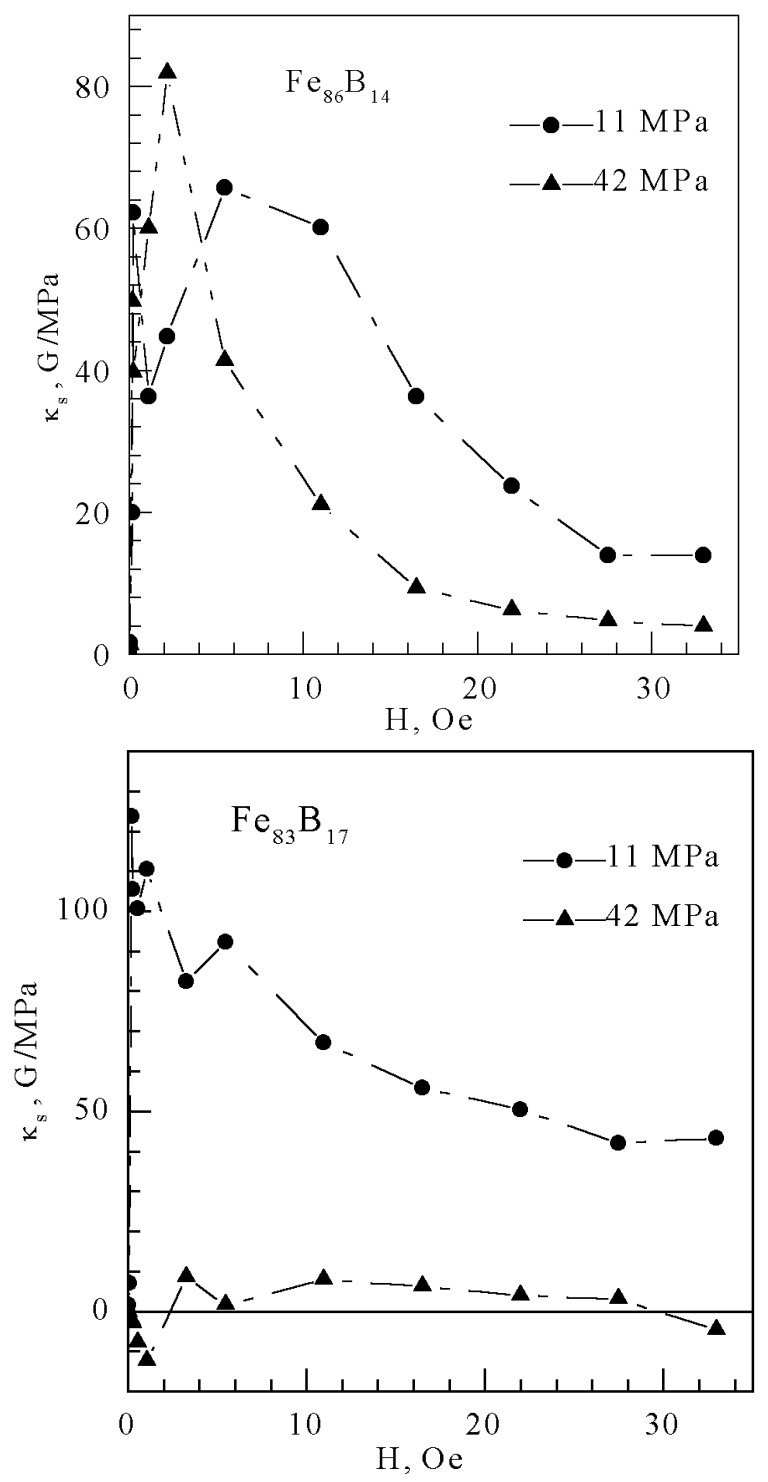

Fig. 7. The magnetostrictive sensitivity $\kappa_{s}$ of the metallic glass $\mathrm{Fe}_{83} \mathrm{~B}_{17}$ (eutectic alloy) and $\mathrm{Fe}_{86} \mathrm{~B}_{14}$ in the whole interval of magnetic fields studied in the tensile stress regions $0-22 \mathrm{MPa}$ $(\langle\sigma\rangle=11 \mathrm{MPa})$ and $22-62 \mathrm{MPa}(\langle\sigma\rangle=42 \mathrm{MPa})$ at room temperature. 


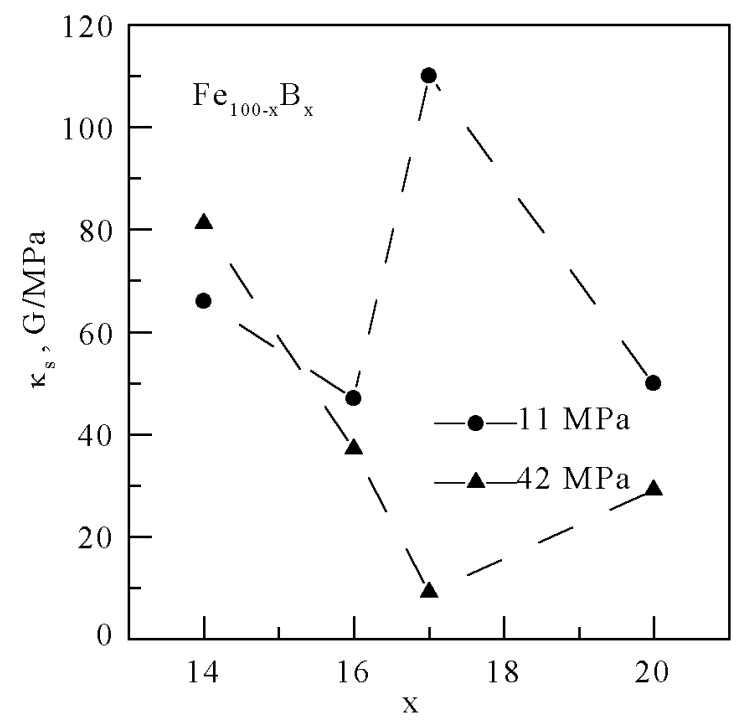

Fig. 8. Magnetostrictive sensitivity $\mathrm{\kappa}_{s}$ of the $\mathrm{Fe}_{100-x} \mathrm{~B}_{x}(x=14$, $16,17,20)$ metallic glasses in the tensile stress regions 0 $22 \mathrm{MPa}(\langle\sigma\rangle=11 \mathrm{MPa})$ and 22-62 $\mathrm{MPa}(\langle\sigma\rangle=42 \mathrm{MPa})$ at room temperature.

\section{Conclusions}

The results obtained confirm that eutectic metallic glass $\mathrm{Fe}_{83} \mathrm{~B}_{17}$ possesses a special electronic structure, which is substantially different from the electronic structure of the other compositions studied. The special electronic structure of the eutectic metallic glass finds reflection in its magnetic properties: this alloy demonstrates the lowest magnetic induction $B$, the lowest magnetic susceptibility $\chi$, and special behavior of magnetostrictive sensitivity $\kappa_{s}$. The magnetostrictive sensitivity of the eutectic metallic glass at low stresses $\kappa_{s}\left(\sigma_{1}\right)$ is much higher than that of all the other metallic glasses studied and is substantially higher than the values $\kappa_{s}\left(\sigma_{2}\right)$ for this alloy at high stresses. The data obtained are in agreement with the data on the lowest values of the Landé $g$ factor and the low-temperature high-field magnetization $M_{s}$ from [3], as well as with the idea of an extremely disordered structure of intercluster boundaries in the eutectic alloy $\mathrm{Fe}_{83} \mathrm{~b}_{17}$, in which the clusters are assumed to have a quasicrystal structure [6].

\section{Acknowledgment}

A. B. B. thanks NATO ASI «Modern Trends in Magnetostriction Study and Application» for hospitality at Kiev in the summer of 2000 .

1. V. Z. Bengus, A. B. Beznosov, V. A. Desnenko, V. V. Eremenko, E. L. Fertman, and E. D. Tabachnikova, Mater. Sci. Forum 2000 343-346, 43 (2000).

2. R. S. Iskhakov, M. M. Karpenko, G. V. Popov, and V. P. Ovcharov, Fiz. Met. Metalloved. 61, 265 (1986).

3. V. Z. Bengus, P. Duhaj, E. B. Korol'kova, E. D. Tabachnikova, A. V. Golik, and S. I. Tarapov, Fiz. Nizk. Temp. 20, 1082 (1994) [Low Temp. Phys. 20, 853 (1994)].

4. A. B. Beznosov, E. L. Fertman, V. A. Desnenko, V. Z. Bengus, and V. A. Ushakov, in: Novye magnitnye materialy mikroelectroniki, MGU, Moskva, 1, 212 (1998) (in Russian).

5. A. B. Beznosov, E. L. Fertman, V. V. Eremenko, V. A. Desnenko, and V. Z. Bengus, Fiz. Nizk. Temp. 25, 857 (1999) [Low Temp. Phys. 25, 641 (1999)].

6. V. S. Kraposhin, Metallovedenie $i$ termicheskaya obrabotka metallov, № 10, 2 (1994) (in Russian).

7. E. Hristoforou and R. E. Reilly, JMMM 119, 247 (1993).

8. S. V. Vonsovsky, Magnetism, Moskva, Nauka (1971) (in Russian).

9. E. S. Borovik and A. S. Milner, Lektsii po ferromagnetizmu, Kharkov, Kharkov State University (1960) (Lectures on the ferromagnetism, in Russian).

10. J. M. Ziman, Principles of the theory of solids, Cambridge, University Press (1972) 